\title{
PUMPING MUSCLES
}

Living cells are surely the smartest of smart materials, and working with them as a plastic medium is often something of a negotiation: they can do a lot by themselves, but only if cajoled in the right way. Cells respond to a range of external stimuli - stem cells, for example, may differentiate in different ways depending on chemical, biochemical, microstructural and mechanical signals from their environment. To make effective use of stem cells for tissue regeneration, therefore, their versatility is simultaneously a virtue and a burden, and their surroundings need to be carefully engineered.

This is illustrated in a study by Sleep et al. on the use of synthetic peptide amphiphiles (PAs) as scaffolds for the in vivo regeneration of muscle ${ }^{1}$. Regrowing atrophied muscle is something mammals are generally fairly good at: muscles contain specialized 'satellite cells', also known as muscle stem cells (MuSCs), that perform this role. But some diseases, notably muscular dystrophies, destroy this capacity, and it also declines with ageing. Samuel Stupp at Northwestern University has been developing PAs as tissue-engineering scaffolds for many years ${ }^{2}$, and the new work in collaboration with stem-cell researcher Helen Blau and her co-workers at the Stanford University School of Medicine demonstrates that these supramolecular systems are now showing clinical promise for muscle growth.

The key attributes of PAs here are that they are biocompatible and selfassembling: they can be injected in solution and will aggregate in vivo with a suitable trigger (for example, chemical, $\mathrm{pH}$ or temperature) into mesoscale gel-like structures with mechanical robustness that can support cells. The diversity of these self-assembled scaffolds is again both an advantage and a challenge: the amphiphilic molecules can aggregate into sheets, micelles, nanofibres, ribbons and other forms, and the factors determining the morphology are not fully understood ${ }^{3}$. Surface charge, hydrophobicity, temperature and chain length may all play a part.

To support MuSCs, Sleep et al. sought long nanofibres with liquidcrystalline alignment, and finding PAs with the right properties was somewhat trial and error. Generically, the PAs have a long-chain aliphatic (palmitoyl) tail and a small peptide head, but even small changes to the amino-acid composition of the latter could produce marked differences in self-assembly. To ensure that the MuSCs proliferate along the correct developmental path, the liquid-crystalline gel has to have just the right stiffness, closely mimicking that of skeletal muscle, and be aligned with the muscle fibres.

Gel formation could be triggered by contact with divalent cations

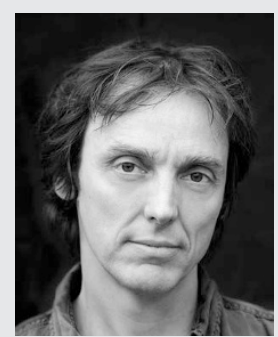

PHILIP BALL

- which, in the form of calcium ions, are conveniently present in vivo. Moreover, the cell growth is promoted by suitable growth factors: proteins that can be injected with and are bound by the self-assembling scaffold. Sleep et al. report that their optimized formulation can support MuSC transplantation and muscle-fibre repair when injected into mice, and that the fibre alignment provides a cue for muscle-cell organization and differentiation that normally requires a force applied to the tissue. The PA scaffold biodegrades in around two weeks - just the right kind of timescale for muscle regrowth. It now remains to be seen if human MuSCs can be similarly persuaded to get to work.

References

1. Sleep, E. et al. Proc. Natl Acad. Sci. USA http://doi.org/10.1073/pnas.1708142114 (2017).

2. Rubert Pérez, C. M. et al. Ann. Biomed. Eng. 43, 501-514 (2001).

3. Fu, I. W., Markegard, C. B., Chu, B. K. \& Nguyen, H. D Langmuir 30, 7745-7754 (2014).

\section{SELF-REPLICATION}

\section{Nanostructure evolution}

DNA origami nanostructures were utilized to replicate a seed pattern that resulted in the growth of populations of nanostructures. Exponential growth could be controlled by environmental conditions depending on the preferential requirements of each population.

\section{Friedrich C. Simmel}

arwinian evolution proceeds through replication of the evolving entities and selection of the 'fitter' - that is, faster replicating - ones over the others. Replication usually occurs with errors, leading to variability and enabling evolutionary change. Over many decades, chemists have attempted to implement these basic processes using synthetic molecules, motivated by both the exploration of potential prebiotic evolutionary scenarios and intellectual curiosity. In many cases, the replicator mechanism was directly inspired by naturally occurring DNA replication, in which the two strands of a double-stranded 